\title{
EVALUACIÓN DE LA ENFERMEDAD DE MEMBRANA HIALINA EN RECIÉN NACIDOS
}

Juan Augusto Perna, MD. *, I’án Pérez H., MD.**, Julia Eugenia Ramírez, MD. **, María Elena Varela D,. MD. **

\section{Resumen}

Para valorar la incidencia de la enfermedad de membrana hialina (EMH), caracterizar la población de riesgo, evaluar el tratamiento instaurado y las complicaciones más frecuentes en recién nacidos, se realizó un estudio retrospectivo descriptivo en los pacientes que ingresaron a la Unidad de Cuidado Intensivo Neonatal (UCIN) del Hospital de San José. Se incluyeron todos los pacientes en los cuales se diagnosticó clínica y paraclínicamente EMH, quienes fueron distribuidos en cuatro grupos según el peso. Los datos recopilados mostraron una mayor incidencia de EMH en los menores de 1.000 g (63\%), en comparación con los mayores de $1.500 \mathrm{~g}(5 \%)$. La complicación encontrada con mayor frecuencia fue la enfermedad pulmonar crónica del recién nacido(24\%), seguida del neumotórax (21\%). En el estudio, la mortalidad fue de $(33,5 \%)$, la cual es mayor a la reportada en la literatura mundial, posiblemente secundaria a la alta incidencia de complicaciones, sobre todo en los pacientes menores de 1.000 g. Finalmente se plantean estrategias de manejo para disminuir la morbimortalidad de los pacientes con EMH.

Palabras claves: Sindrome de distrés respiratorio del recién nacido, enfermedad de membrana hialina, surfactante pulmonar, cuidado intensivo neonatal, prematuro.

\section{Introducción}

El síndrome de distrés respiratorio (SDR) del recién nacido o enfermedad de membrana hialina es un déficit de surfactante pulmonar, característico de la prematurez o secundario al consumo del mismo; es una de las entidades mas diagnosticadas en recién nacidos pretérmino (RNPT). El advenimiento de nuevas técnicas de cuidado intensivo neonatal ha permitido un incremento marcado en la supervivencia de los prematuros muy pequeños, con el subsiguiente aumento de la morbilidad y la disminución de la mortalidad por esta causa.

La presente investigación, realizada en la Unidad de Cuidado Intensivo Neonatal (UCIN) del Hospital de San José, tiene como objetivos: caracterizar la población de recién nacidos pretérmino de alto ries-

\footnotetext{
Pediatra. Unidad de Cuidado Intensivo Neonatal. Hospital de San José.

** Residente de III año, Departamento de Pediatria, Fundación Universitaria de Ciencias de la Salud. Hospital de San José. Bogotá D),C.. Colombia.
}

go; determinar los tratamientos instaurados, las complicaciones asociadas con mayor frecuencia y los gérmenes más frecuentemente aislados, así como hacer una revisión de los nuevos conceptos en cuanto a factores de riesgo, físiopatología y tratamiento, tanto preventivo como terapéutico. Con base en esta información, los autores plantearon diferentes estrategias para disminuir la morbimortalidad, reducir la estancia hospitalaria, mejorar la calidad de vida y disminuir el costo emocional para las familias y los costos económicos para las entidades de salud.

\section{Generalidades}

El SDR consiste en la dificultad respiratoria secundaria a la incapacidad del neumocito tipo II para sintetizar surfactante pulmonar, lo que ocasiona disminución del volumen pulmonar y colapso alveolar progresivo.' Es característico de los neonatos prematuros y de bajo peso (menores de $.500 \mathrm{~g})^{2}$ y esto lo diferencia de la defíciencia secundaria de surfactante por consumo, que corresponde al síndro- 
me de distrés respiratorio adulto neonatal, característico del recién nacido a término. ${ }^{3}$ Desde la introducción, en 1980, del surfactante exógeno para la prevención y tratamiento de esta enfermedad, el curso de la misma, en cuanto a morbilidad y mortalidad, ha cambiado sustancialmente.

La incidencia de enfermedad de membrana hialina se incrementa a medida que disminuye la edad gestacional; es así que en los infantes de 29 semanas, la incidencia asciende a $50 \%$, mientras que es de apenas $5 \%$ entre los recién nacidos de 34 semanas de gestación. ${ }^{4}$ Por otra parte, según datos correspondientes a Estados Unidos, en 1995 la mortalidad por esta enfermedad entre los recién nacidos prematuros, con un peso comprendido entre 500 y $1.500 \mathrm{~g}$, fue de $20 \%$. $^{5,6,7}$

Los factores de riesgo que predisponen a la presentación del SDR comprenden:

1. La prematurez y el bajo peso al nacer: hay una proporción de uno a uno entre el surfactante activo $50 \%$ y el inactivo $50 \%$. En el recién nacido prematuro es más rápido el paso del surfactante activo a inactivo. ${ }^{1}$

2. La cesárea electiva sin trabajo de parto: el trabajo de parto estimula la liberación de catecolaminas que a su vez induce la secreción de surfactante pulmonar. ${ }^{2}$

3. La asfixia perinatal: debido al consumo de surfactante endógeno y a la disminución de la recaptación del mismo. ${ }^{1,2,3}$

4. El género masculino: los andrógenos causan una deficiencia en la maduración pulmonar, relacionada con una menor síntesis de surfactante por parte de los neumocitos tipo II. Por esto, la incidencia de EMH es mayor en varones pretérmino. ${ }^{4}$

5. La raza: es más común en sujetos de raza blanca que en negros, porque la maduración fetal del surfactante pulmonar es más lenta en los primeros que en los segundos. En la actualidad, gracias al uso del surfactante exógeno, la mortalidad entre los prematuros de raza blanca ha disminuido en $41 \%$ y entre $6 \%$ y $8 \%$ en los de raza negra. De otra parte, en los niños la evolución es más benigna. ${ }^{8}$

6. El segundo de los gemelos: las gestaciones múltiples están relacionadas con concentraciones séricas más bajas de corticoides en las madres, que los embarazos únicos..$^{8,9,10}$

7. El antecedente de recién nacido anterior con EMH. ${ }^{1}$

8. La diabetes materna: este es un factor sugerido, pues la diabetes en la madre parece inducir una disminución del contenido de proteínas del surfactante y la disfunción del mismo., 2,3,4

Por otra parte, ciertas enfermedades y condiciones maternas pueden disminuir el riesgo de EMH tales como: la hipertensión inducida por el embarazo, la hipertensión crónica, el abruptio de placenta subagudo, la adicción a narcóticos, el consumo de cigarrillos, la ruptura prematura de membranas y las infecciones congénitas. ${ }^{1,4}$

La secreción de surfactante, que ocurre con la primera respiración al nacer, probablemente resulta de la asociación de múltiples estímulos que combinan los efectos de las concentraciones elevadas de catecolaminas y la expansión pulmonar. ${ }^{7}$

El surfactante pulmonar es una sustancia característica de los mamíferos, en cuya composición intervienen fosfolípidos (70\%-90\%), lípidos neutros y colesterol (10\%) y proteínas $(10 \%))^{3}$ su función es disminuir la tensión superficial en el alveolo, evitando el colapso de esta estructura durante el proceso de la respiración. El surfactante es producido por los nemocitos tipo II, en forma de cuerpos lamelares, estructuras que aparecen a las 20 semanas de edad gestacional, pero que requieren un proceso de maduración iniciado luego de las 26 a 28 semanas (Figura 1).

La fase inicial en la síntesis de los fosfolípidos del surfactante compromete la fabricación del ácido fosfatídico. La fosfatidilcolina es el componente más importante del surfactante, tanto en función como 


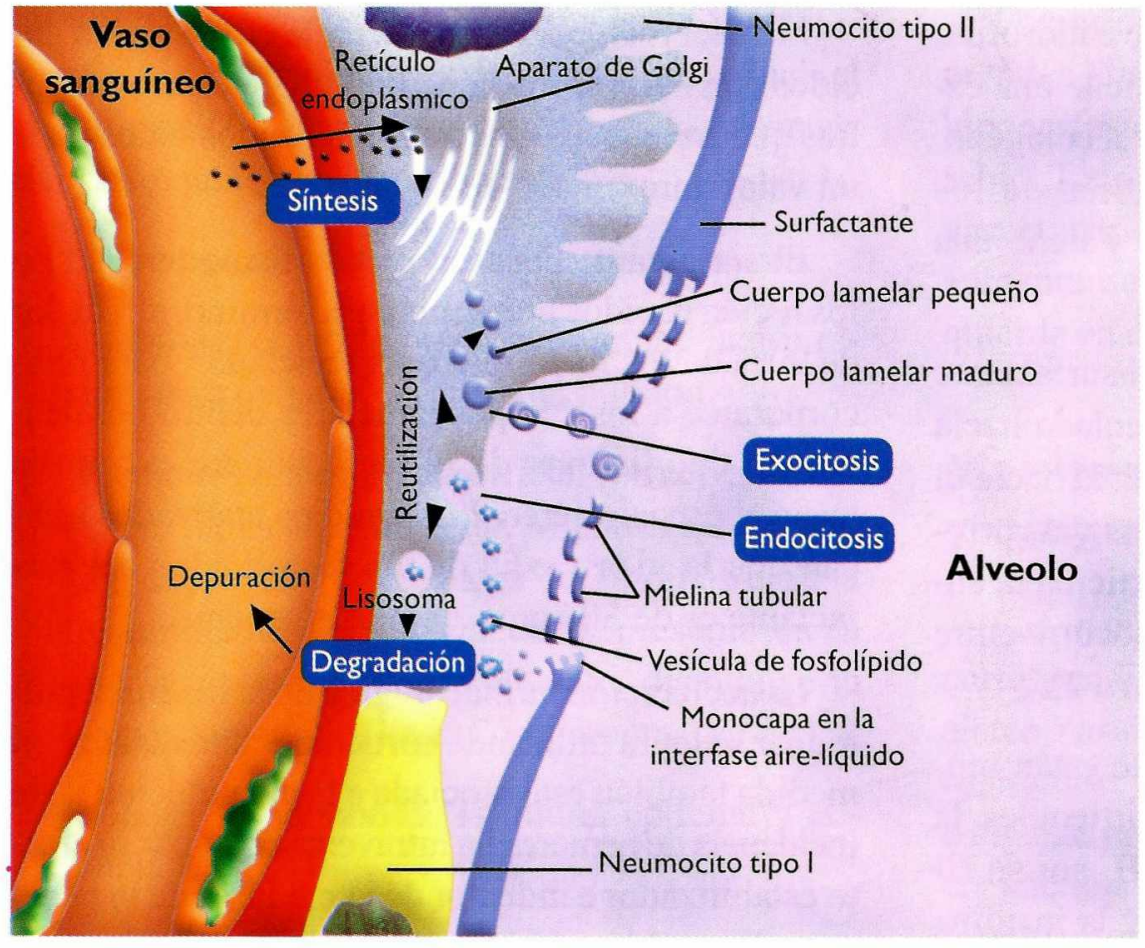

Figura 1. Síntesis, reutilización y degradación del surfactante por los neumocitos tipo II de las paredes alveolares. lamelares. Al final termina formando un polímero similar al colágeno, con una triple hélice y un peso molecular de $650 \mathrm{Kda} \cdot{ }^{12,13} \mathrm{Se}$ ha demostrado, in utero, que es capaz de aumentar la concentración de fosfolípidos e inhibir la secreción del surfactante a partir del neumocito tipo II, gracias a la activación de receptores dependientes de este mecanismo $^{12}$ por lo que parece actuar como regulador del metabolismo del surfactante; in vitro, activa los macrófagos contra infecciones por virus de la influenza, Staphylococcus aureus, Streptococcus pneumoniae $^{I t, 15}$ y virus herpes simple tipo I. ${ }^{16}$ Hasta la fecha no se han identificado pacientes con deficiencia de esta proteína.

Las proteínas B y C (SP-B y SP-

en cantidad, pues constituye entre $60 \%$ y $65 \%$ del mismo; es el fosfolípido más abundante $(80 \%)^{10} \mathrm{y}$ se encuentra en dos formas: saturada y no saturada. La saturación es el resultado de la unión al ácido palmítico, ya sea en el carbono 16 o en el carbono 2 de la cadena central; esta última unión permite la formación de una doble banda que facilita la función de la interfase aire-líquido; por su parte, la forma no saturada es inactiva y no tiene ninguna relación con el metabolismo del surfactante.

El fosfatidilglicerol es un fosfolípido ácido y constituye de $4 \%$ a $15 \%$ del contenido lipídico del surfactante; aunque no es necesario para la función normal de esta sustancia, es un inductor fiable de la madurez fetal, pues aparece a partir de la semana 25-26 de la gestación, junto con el fosfatidilinositol. ${ }^{10,11,12}$ Además de las moléculas mencionadas, otros fosfolípidos constituyentes son la esfingomielina y la etanolamina.

En cuanto al contenido proteínico, hay al menos cuatro proteínas específicas reconocidas con las letras A, B, C y D. La proteína A (SP-A) es hidrofílica y tiene una síntesis de novo fuera de los cuerpos
C) son hidrófobas y constituyen de $2 \%$ a $4 \%$ de los componentes del surfactante. ${ }^{12}$ La proteína $\mathrm{B}$ es codificada por un gen del cromosoma 2; una vez secretada por los neumocitos tipo II puede ser recaptada y concentrada junto con fosfolípidos en los cuerpos lamelares y de esta manera promueve la maduración de estos. ${ }^{16}$. La proteína B es necesaria para la formación de la mielina tubular ${ }^{15} \mathrm{y}$ es vital para las propiedades tensoactivas del surfactante, ya que facilita la absorción de los fosfolípidos en la superficie alveolar. La ausencia congénita de proteína B ocasiona un SDR severo, desde el nacimiento y causa la muerte un uno o dos días; esta alteración es más común en los recién nacidos a término. ${ }^{17,18}$

La proteína $C$ es codificada en el cromosoma 8 y se produce tempranamente durante el desarrollo. Contribuye a la maduración del recién nacido a término y se piensa que la ausencia del ácido ribonucleico mensajero (RNA-m) correspondiente causa la detención del desarrollo pulmonar. La función de esta proteína es facilitar la absorción de fosfolípidos en la superficie alveolar ${ }^{19} \mathrm{y}$ su deficiencia congénita no ha sido determinada en humanos. 
La proteína D (SP-D) es hidrosoluble e hidrófíla, está codificada en el cromosoma 10 y tiene una estructura similar a la proteína A (SP-A) y al colágeno. Está presente en los gránulos secretores de los neumocitos tipo II y las células claras y tiene una función defensiva. ${ }^{2,3,17}$

Desde el punto de vista molecular, el surfactante tiene dos porciones, una hidrófoba orientada hacia la luz del alveolo y otra hidrófíla orientada hacia el tejido alveolar. ${ }^{5} \mathrm{Su}$ síntesis involucra dos vías principales, de las cuales aquella en que participa la enzima metiltransferasa, es más precoz y ocurre entre las 22 y 24 semanas de gestación (Figura 2). ${ }^{5}$

La síntesis y secreción de surfactante están moduladas por diversos factores; así, los corticoides, la hormona liberadora de tirotropina (TRH, por su sigla en inglés), las hormonas tiroideas y la insulina fomentan la síntesis, mientras que el trabajo de parto, los beta agonistas, la aminofilina, las purinas y las prostaglandinas promueven la liberación.,
La reservas $(p o o l)$ de surfactante en los recién nacidos con SDR son de 2 a $10 \mathrm{mg} / \mathrm{Kg}$ de peso, mientras que en los recién nacidos a términos sanos tiene un valor aproximado de $100 \mathrm{mg} / \mathrm{Kg} .{ }^{25}$

El surfactante tiene un metabolismo lento. ${ }^{26}$ En los recién nacidos pretérmino y a término, alrededor de $90 \%$ de los fosfolípidos es reciclado ${ }^{27}$ para reincorporarse a los cuerpos lamelares dentro del neumocito tipo II, antes de su secreción por exocitosis hacia el espacio aéreo. Los estudios realizados indican que la vida media de los fosfolípidos del surfactante es de 30 horas aproximadamente.

La aceleración farmacológica de la madurez pulmonar se logra utilizando corticoides antenatales; al medida también está asociada a la disminución de la incidencia de hemorragia intraventricular por su efecto estabilizador e inductor de la madurez de la pared vascular en la matriz germinal. 9,28,29

La eficacia de los corticoides ha sido comprobada en numerosos estudios y no se ha demostrado que

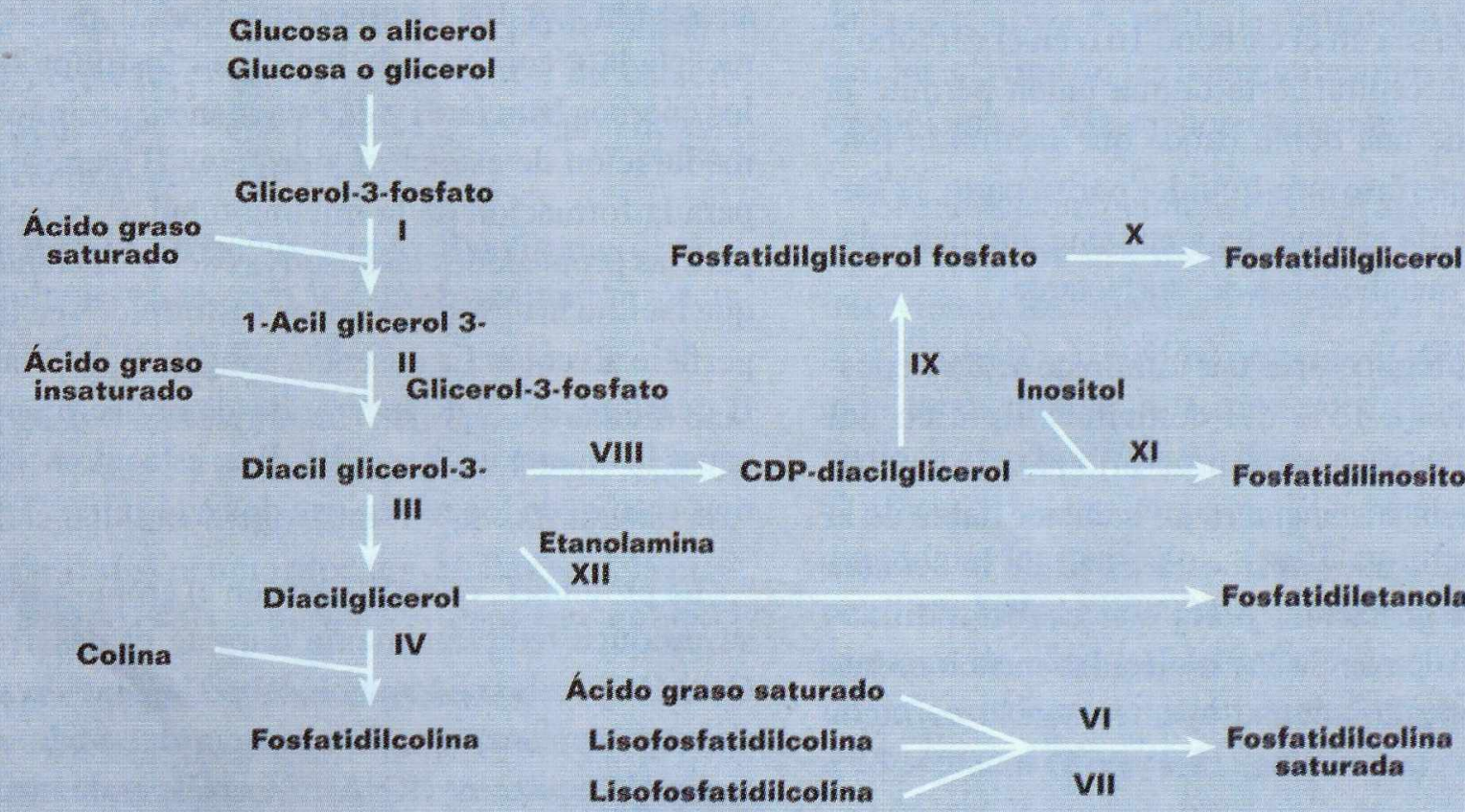

Figura 2. Ciclo bioquímico de la formación del surfactante pulmonar. Tomado de Martin R, Medicina Neonatal y Perinatal, Sexta Edición, Editorial Mosby, 1.998, Pág 1000. 
su utilización aumente la frecuencia de infecciones; $;^{30,31}$ es más, también son efectivos para reducir el riesgo de SDR cuando son administrados después del parto, junto con el surfactante. ${ }^{31}$

Las principales funciones del surfactante son:

1. Estabilizar el tejido pulmonar durante la espiración, para evitar el colapso del pulmón.

2. Disminuir la tensión superficial (o fuerza de cohesión molecular) alveolar. Mientras que la tensión superficial del agua es de 72 dinas/cm., la fosfatidilcolina del surfactante, presente en la interfase aire-líquido del alveolo, disminuye la tensión superficial hasta $25 \mathrm{dinas} / \mathrm{cm}$.

3. Proteger al pulmón de la injuria epitelial y endotelial.

\section{Proveer defensas contra la infección.}

Para evitar el colapso pulmonar se necesita mínimo una presión de $2 \mathrm{~cm}$ de agua (Figura 3 ).

Al microscopio, el pulmón del paciente con SDR luce muy similar al tejido hepático; hay zonas de atelectasia, con pocos alvéolos dilatados y una membrana eosinofílica hialina localizada en la superficie de contacto con el aire de los bronquiolos terminales y en los conductos alveolares. Cuando se regeneran las células alveolares tipo II, aumenta la síntesis del surfactante y se controla la enfermedad.
Los principales cambios fisiopatológicos comprenden hipoxemia secundaria y acidosis láctica por hipoperfusión y predominio del metabolismo anaerobio. Todo ello lleva a hipoperfusión pulmonar por vasoconstricción, lo que genera más hipoxemia y aumento del cortocircuito (shunt) de derecha a izquierda en el ductus arterioso y en el foramen oval.

\section{Manifestaciones clínicas y dîagnóstico}

Las manifestaciones clínicas de la EMH son las correspondientes a la dificultad respiratoria, como aleteo nasal, taquipnea, tirajes, cianosis y quejido espiratorio, las cuales aparecen desde el nacimiento o algunas horas después. Son signos de mal pronóstico: ictericia precoz y progresiva, edema generalizado blando o duro (escleroderma), oliguria, hipotonía, episodios de apneas y episodios con convulsiones secundarias a hipoxia. Puede presentarse hemorragia intracraneana, además de hemorragia pulmonar masiva con desenlace fatal.

En los estudios radiológicos pueden evidenciarse infiltrados típicos, difusos, retículonodulares, simétricos y bilaterales, con broncograma aéreo. El infiltrado nodular es secundario al colapso alveolar y el reticular al edema pulmonar; a su vez, el broncograma aéreo muestra los bronquiolos hiperinsuflados que llegan a alvéolos atelectásicos y como rasgo característico, el infiltrado predomina en las áreas superiores. La extensión del infiltrado permite hacer una clasificación en cuatro grados: 2,3,32

- Grado I: Infiltrados parahiliares bilaterales característicos, que no borran la silueta cardíaca.

- Grado II: Infiltrados que borran la silueta cardíaca y presenta broncograma aéreo.

- Grado III: Compromete hasta los dos tercios del parénquima pulmonar, más lo anterior
Figura 3. Esquema de la ecuación de LaPlace. $\mathrm{P}=2$ TS $($ dinas $/ \mathrm{cm}) \times 10 / \mathrm{r}$. P $=$ presión en $\mathrm{cm}$ de agua. $\mathrm{TS}=$ tensión superficial. $\mathrm{r}=$ radio en micrómetros. 
- Grado IV: Infiltrados que cubren todo el parénquima pulmonar hasta el tercio externo, como "vidrio esmerilado".

Es importante recordar que a mayor grado radiológico mayor severidad de la enfermedad. Además, no se puede diferenciar radiológicamente el SDR de una neumonía intrauterina, cuyo germen etiológico más común es el estreptococo del grupo B lo que explica y justifica el uso de antibióticos en algunas ocasiones. $^{33}$

La ventilación mecánica disminuye el curso de la enfermedad y aumenta la supervivencia, pero también eleva la incidencia de complicaciones. Al respecto, las complicaciones asociadas con mayor frecuencia son: neumotórax, neumomediastino, hemorragia pulmonar, displasia broncopulmonar y persistencia del ductus. ${ }^{3}$

Los estudios paraclínicos comprenden:

a. Gases arteriales. ${ }^{3}$

b. Cuadro hemático con VSG.

c. Electrolitos y glucemia. ${ }^{9}$

d. Estudio de sepsis.

e. Ecocardiograma: descarta la presencia o persistencia del ductus arterioso, como factor que podría afectar la función pulmonar y permite detectar hipertensión pulmonar.

En el diagnóstico diferencial hay que considerar neumonía, taquipnea transitoria, cardiopatías complejas, edema pulmonar y síndrome de distrés adulto neonatal.

\section{Tratamiento}

El manejo del SDR del recién nacido involucra medidas generales y específicas. Las primeras comprenden el estricto monitoreo hemodinámico y respiratorio, junto con accesos venosos adecuados, la termorregulación, el balance nutricional y de fluidos, la administración de antibióticos sistémicos y la transfusión.

Es importante mantener una frecuencia cardiaca adecuada, así como una tensión arterial medial (TAM) entre 50 y $80 \mathrm{~mm}$ de $\mathrm{Hg}$ ) (según edad y peso), un $\mathrm{pH}$ mayor de 7,25 y una presión arterial $(\mathrm{Pa}) \mathrm{de}$ $\mathrm{CO}_{2}$ entre 45-55 mm de $\mathrm{Hg}$ (bien correlacionada con el $\mathrm{pH}$ ). Las presiones altas de $\mathrm{CO}_{2}$ disminuyen el consumo metabólico de $\mathrm{O}_{2}$, producen vasodilatación cerebral, aumentan el flujo cerebral, disminuyen el volutrauma y el barotrauma. ${ }^{34}$ De igual modo, es fundamental mantener al paciente monitorizado con un oxímetro de pulso y el valor ideal es de $90 \%$, que corresponde a una $\mathrm{PaO}_{2}$ de $80 \mathrm{~mm}$ de $\mathrm{Hg}$. ${ }^{3,34}$

Los niños con SDR requieren un ambiente térmico óptimo para minimizar el consumo y los requerimientos de oxígeno $\left(37^{\circ} \mathrm{C}\right)$, pues el estrés causado por el frío lleva a un incremento de la tasa metabólica lo que produce hipoxia secundaria. ${ }^{2,3}$

Es importante proveer un adecuado aporte calórico en el infante con insuficiencia respiratoria, incluso con la utilización de nutrición parenteral. Para un aporte adecuado de glucosa, aminoácidos y lípidos en los prematuros (especialmente en menores de $1.000 \mathrm{~g}$ ) se recomiendan alrededor de $60 \mathrm{kcal} / \mathrm{kg} / \mathrm{día}$, de las cuales $10 \%$ deben ser proteínas, para mantener un balance nitrogenado positivo. Puede ser beneficioso ofrecer volúmenes pequeños de leche materna por gavaje, una vez se estabiliza el estado respiratorio, incrementados progresivamente según la tolerancia. El estímulo enteral de $0,5 \mathrm{cc}$ de leche materna cada tres horas evita la atrofia de las microvellosidades intestinales y estimula la liberación de hormonas gastrointestinales. 3,36,37

Es aconsejable no exceder el aporte hídrico pues está demostrado que ello favorece el riesgo de persistencia del ductus arterioso, la enterocolitis necrotizante e incluso la displasia broncopulmonar. ${ }^{3}$ Además, hay que mantener una diuresis mínima de $0,5-1 \mathrm{cc} / \mathrm{kg} /$ hora, para lo cual puede utilizarse furosemida, a dosis de $1 \mathrm{mg} / \mathrm{kg} /$ día 2 veces al día.

La administración de antibioticoterapia específica es necesaria, puesto que resulta difícil el diagnóstico diferencial con la neumonía in utero por estreptococo beta hemolítico del grupo B, infección que es potencialmente fulminante en los prematuros. ${ }^{37}$

Las transfusiones están indicadas para mantener una adecuada capacidad de transporte de oxígeno se 
recomienda un hematocrito venoso mayor de $40 \%$ durante la fase aguda. Al disminuir los requerimientos de la fracción inspirada de oxígeno $\left(\mathrm{FIO}_{2}\right)$, disminuye la incidencia de retinopatía del prematuro, porque mejora la afinidad del oxígeno por la hemoglobina. Es conveniente el uso de eritropoyetina recombinante humana a dosis de $250 \mathrm{u} / \mathrm{kg} 3$ veces a la semana, desde el tercer día de vida al cuadragésimo segundo día de vida, o en pacientes de muy bajo peso (menores de $1.500 \mathrm{~g}$ ), o en menores de $33 \mathrm{se}-$ manas. El uso de esta hormona disminuye en $50 \%$ la necesidad de transfusiones sanguíneas. ${ }^{38}$

\section{Manejo específico}

\section{Esteroides antenatales}

La aceleración farmacológica de la madurez pulmonar se logra utilizando corticoides antenatales, medida que, además, está asociada a la disminución de la incidencia de hemorragia intraventricular por el efecto estabilizador e inductor de la madurez de la pared vascular en la matriz germinal que tienen tales compuestos. Los corticoides disminuyen la incidencia y severidad de la EMH y se recomiendan desde la semana 24 hasta la 34, e incluso en todas las madres con amenaza de parto prematuro (por ruptura prematura de membranas, hipertensión inducida por el embarazo, infecciones, niños grandes, embarazos gemelares, madres con tocolíticos). ${ }^{3}$ El tratamiento consiste en betametasona $12 \mathrm{mg}$ IM cada 12 horas, para una dosis total de $24 \mathrm{mg}$ y parto en los siguientes siete días. ${ }^{28}$ También es aceptable emplear cuatro dosis de dexametasona ( $6 \mathrm{mg}$ cada 12 horas) por 4 dosis. Los corticoides deben administrarse a la madre por lo menos entre 24 y 48 horas antes del parto, porque la terapia con menos de 24 horas no es efectiva. ${ }^{2,7}$

\section{Surfactante pulmonar}

Desde la descripción inicial de la deficiencia de surfactante hecha por Avery en 1959, como causa de la EMH, se ha progresado mucho en la comprensión de los procesos de síntesis, secreción, ultraestructura y función del surfactante pulmonar. Al comienzo, se utilizó un preparado japonés desarrollado por Fujiwara ${ }^{37,39}$ compuesto de fosfolípidos aerolizados; luego, Hallman empleó un derivado de surfactante humano extractado del líquido amniótico de recién nacidos a término, ${ }^{39}$ pero no fue utilizado por razones éticas. Esto explica las diferencias existentes entre los surfactantes naturales (que contienen apoproteínas) y cuya acción es más rápida y sostenida, respecto a los artificiales, cuya bioactividad y dispersión se facilita por la adición de alcoholes, como hexadecanol y tiloxapol (Tabla 1). ${ }^{3,33,35,40}$

Las dos modalidades de aplicación son la de rescate y la profilaxis. La primera implica la administración de surfactante, una vez confirmada por radiología la presencia de EMH, junto con requerimientos de oxígeno mayores de $30 \% .^{11,32,41}$ Por su parte, la profilaxis implica la administración de surfactante inmediatamente después del nacimiento (minutos después del nacimiento) y los mejores resultados en esta modalidad se dan en prematuros menores de 29 a 30 semanas, por debajo de $1.250 \mathrm{~g}$. Por encima de estos límites la relación riesgo/beneficio no justifica la utilización del surfactante. Al igual que en la modalidad de rescate, la terapia disminuye la severidad y mortalidad de la EMH, mejora la supervivencia a los 28 días sin displasia broncopulmonar $\mathrm{y}$, según algunos estudios, disminuye la incidencia de hemorragia intraventricular severa y retinopatía,

\begin{tabular}{|c|c|c|c|}
\hline $\begin{array}{l}\text { Nombre } \\
\text { genérico }\end{array}$ & $\begin{array}{l}\text { Nombre } \\
\text { comercial }\end{array}$ & Preparación & Fabricante \\
\hline Beractant & Survanta & $\begin{array}{l}\text { Extracto de pulmón } \\
\text { bovino + DPPC + } \\
\text { Tripalmitina + ácido } \\
\text { palmítico. }\end{array}$ & $\begin{array}{c}\text { Abbott } \\
\text { Laboratories } \\
\text { (Estados Unidos) }\end{array}$ \\
\hline $\begin{array}{l}\text { Surfactante } \\
\text { porcino }\end{array}$ & Curosurf & $\begin{array}{l}\text { Extracto de pulmón } \\
\text { porcino + cloroformo } \\
+ \text { metanol }\end{array}$ & $\begin{array}{c}\text { Chiesi } \\
\text { Pharmaceuticals } \\
\text { (Italia) }\end{array}$ \\
\hline $\begin{array}{l}\text { Colfosceril } \\
\text { palmitato, } \\
\text { hexadecanol, } \\
\text { tiloxapol } \\
\text { (CPHT) }\end{array}$ & Exosurf & $\begin{array}{c}\text { DPPC + Hexadecanol } \\
\text { al } 9 \%+\text { Tiloxapol } \\
\text { al } 6 \%\end{array}$ & $\begin{array}{l}\text { Burroughs } \\
\text { Wellcome } \\
\text { (Estados Unidos) }\end{array}$ \\
\hline
\end{tabular}


El surfactante pulmonar se administra por infusión en bolos, por tubo orotraqueal de la siguiente manera:

Dosis:

- Survanta®: $100 \mathrm{mg} / \mathrm{kg}$ de peso $(4 \mathrm{cc} / \mathrm{kg})$

- Exosurf® $67,5 \mathrm{mg} / \mathrm{kg}(5 \mathrm{cc} / \mathrm{kg})$

- Curosurf® 100 a 200mg/kg $(1 \mathrm{cc}=80 \mathrm{mg})$

Merece la pena señalar que la aplicación de surfactante asociada al uso de esteroides logra un efecto aditivo en la prevención del SDR. ${ }^{41}$ Es aconsejable incrementar la frecuencia ventilatoria y $\mathrm{la} \mathrm{FIO}_{2}$ un $10 \%$ durante su aplicación. La dosis total se divide en cuatro cuadrantes que se aplican por gravedad (cambios posturales), con la utilización del Survanta®.

Las complicaciones que pueden presentarse durante el procedimiento incluyen bradicardia, desaturación, obstrucción del tubo, aplicación selectiva y aumento del barotrauma. 2,3,40,41

Al mejorar la distensibilidad, es aconsejable disminuir las presiones ventilatorias y el $\mathrm{FIO}_{2}$ en forma progresiva y, luego de la aplicación, es necesario vigilar la aparición de signos de reapertura del ductus, hemorragia pulmonar o neumotórax.

Los criterios para redosificación, señalados hasta la fecha incluyen el requerimiento de ventilación mecánica con presiones medias mayores de 7-8 cm de agua y $\mathrm{FIO}_{2}$ superiores a $30 \%-40 \%$. Es posible colocar hasta cuatro dosis con intervalos de seis a ocho horas y los reportes muestran que las dosis adicionales (dos y tres) disminuyen la severidad de la EMH y la mortalidad asociada. ${ }^{11}$

\section{Ventilación asistida}

Los requerimientos de ventilación asistida en el manejo de la EMH son: $\mathrm{FIO}_{2}$ entre 90 y $100 \%$; PIM entre 25 y $35 \mathrm{~cm}$ de agua; PEEP entre 4 y 6 ; relación inspiración: espiración (I:E) de 1:2 o de 1:1,5; IMV entre 40 y 60/min; presión media de la vía aérea entre 4 y $6 \mathrm{~cm}$ de agua; volumen corriente entre 3 y 4 $\mathrm{cc} / \mathrm{kg}$ y flujo entre 4 y 6 litros. $^{42}$

\section{Presión positiva continua al final de} la vía aérea

La presión positiva continua al final de la vía aérea (CPAP) aumenta los volúmenes alveolares y promueve el reclutamiento y la estabilidad alveolar, junto con la redistribución del líquido pulmonar, lo que incrementa la capacidad residual funcional $\mathrm{y}$, a su vez, la oxigenación. Como tratamiento, disminuye los requerimientos de oxígeno y la ventilación mecánica pero aumenta el barotrauma. Debe utilizarse de manera temprana, con $\mathrm{FIO}_{2}$ mayores de $40 \%$ y $\mathrm{PaO}_{2}$ menores de $50 \mathrm{~mm}$ de $\mathrm{Hg}$. ${ }^{41,42}$

\section{Óxido nítrico}

El óxido nítrico se ha utilizado en infantes a término con hipertensión pulmonar, a dosis entre $5 \mathrm{y}$ 20 partes por millón, con niveles estrictos de metahemoglobina y dióxido nítrico. ${ }^{43,44}$

\section{Oxigenación por membrana extracorpórea}

La técnica de oxigenación por membrana extracorpórea (ECMO, por su sigla en inglés) se utiliza sólo en niños mayores de $2 \mathrm{~kg}$. por el alto riesgo de hemorragia intraventricular en los menores de este peso. La supervivencia asociada al uso de ECMO en niños con casos severos de EMH es de $84 \%$.

\section{Ventilación de alta frecuencia}

En algunos estudios se demostró que los pacientes tratados con ventilación de alta frecuencia tuvieron menos dependencia de oxígeno y requerimientos inferiores de ventilación mecánica, así como una menor incidencia de displasia broncopulmonar (DBP) ${ }^{45}$

\section{Esteroides postnatales}

El uso de esteroide postnatales está indicado en forma temprana (menos de 96 horas de vida) y se considera que tardíamente (más de tres semanas̊) no disminuye el riesgo de displasia broncopulmonar y, en cambio, aumenta los efectos secundarios como la hemorragia de vías digestivas altas, la hipertensión arterial, la hiperglucemia y la supresión del eje hipotálamo- hipófisis-suprarrenal. Esta medida es re- 
comendable en pacientes ventilados entre el séptimo y el decimocuarto día de vida postnatal y se hace con dexametasona a dosis de 0,2 a $0,5 \mathrm{mg} / \mathrm{kg} / \mathrm{dí}$, al comienzo, para disminuir luego un tercio de la dosis cada tercer día, hasta suspender por completo. ${ }^{46,47}$

\section{Vitamina A (retinol)}

En la DBP la vitamina A juega un papel importante en la regeneración del epitelio pulmonar. El esquema indicado para la aplicación de vitamina $\mathrm{A}$ es por vía IM, 14 veces en días alternos a dosis de $2.000 \mathrm{UI} / \mathrm{kg} /$ dosis o por vía enteral $(4.000 \mathrm{UI} / \mathrm{kg} /$ día), a partir del décimo día postnatal. ${ }^{41}$

\section{Vitamina $E$}

La vitamina $E$ es un antioxidante que se ha empleado en diferentes estudios, sin mostrar beneficios. ${ }^{41}$

\section{Superóxido dismutasa}

El uso de superóxido dismutasa, un antioxidante que se puede administrar por vía intratraqueal, está en investigación como método para disminuir la incidencia de DBP. También se han realizado estudios con ácidos grasos poliinsaturados (Intralipid®), cuyos resultados muestran que la administración muy temprana, por vía intravenosa, puede aumentar las presiones en la arteria pulmonar, incrementar la dependencia al ventilador y potencializar la displasia broncopulmonar. ${ }^{41}$

\section{Materiales y métodos}

Se realizó un estudio descriptivo y retrospectivo de las historias clínicas de la Unidad de Recién Nacidos del Hospital de San José, desde enero de 1999 hasta octubre de 2000. Fueron incluidos aquellos pacientes que cumplieron los criterios clínicos, paraclínicos y radiológicos de enfermedad de membrana hialina.

Las variables consideradas incluyeron las siguientes:

1. Recién nacido pretérmino: todo paciente que nace después de la semana24 y antes de la semana 38 de gestación. ${ }^{3}$
2. Enfermedad pulmonả crónica: patología resultante de la dependencia de $\mathrm{O}_{2}$ suplementario después de los 28 días de vida y el hallazgo de un parénquima pulmonar anómalo en la radiografía del tórax. También se consideró como patología presente en el recién nacido menor de 30 semanas de gestación o un peso menor de $1.500 \mathrm{~g}$., con necesidad de $\mathrm{O}_{2}$ suplementario después de las 36 semanas de edad post-concepcional. ${ }^{41,47,48}$

3. Retinopatía de la prematuridad: enfermedad retiniana vasoproliferativa y multifactorial, cuya incidencia aumenta con la edad gestacional decreciente y relacionada directamente con el uso de altas concentraciones de oxigeno. ${ }^{49}$

4. Hemorragia intracraneana: sangrado extracerebral en los espacios epidural, subdural o subaracnoideo, en el parénquima cerebral o cerebeloso, o en los ventrículos, a partir de la matriz germinal subependimaria o de los plexos coroideos. ${ }^{50,51}$

5. Días de ventilación mecánica: considera las 24 horas del día o fracciones de día del paciente conectado a un ventilador mecánico.

6. Días de estancia hospitalaria: es decir, 24 horas, o fracciones de día, del paciente dentro de la Institución.

7. Muerte: cesación o término de la vida; ausencia de signos vitales y que no responde a maniobras de reanimación. ${ }^{52}$

8. Neumotórax: enfermedad producida por la entrada de aire exterior o pulmonar en el espacio intrapleural. $^{51}$

9. Ductus arterioso persistente (DAP): Permeabilidad del conducto que comunica la aorta descendente con la arteria pulmonar; dicha estructura está presente en la circulación fetal para favorecer el flujo sanguíneo cuando hay aumento de las resistencias pulmonares. ${ }^{51}$

10.Hemorragia pulmonar: presencia de hematíes en los espacios aéreos, espacios intersticiales o ambos, como consecuencia de la extravasación sanguínea en los capilares pulmonares. ${ }^{51}$ 


\section{Resultados}

La incidencia de EMH durante los meses de enero de 1999 a octubre de 2.000 ( 22 meses) fue de $9 \%$, que correspondió a 150 pacientes de un total de 1.794 menores ingresados a la Unidad de Recién Nacidos del Hospital de San José, en la ciudad de Bogotá (Tabla 2).

La incidencia más alta se observó en pacientes menores de $1.000 \mathrm{~g}$., con una cifra de $63 \%$, correspondiente a 31 pacientes de los 49 ingresados. En los neonatos con un peso comprendido entre 1.001 gr.- y 1.250 g tal parámetro fue de $29 \%$ ( 23 pacientes de 79 ingresados), un $19 \%$ en los niños con un peso de $1.251 \mathrm{gr}$. a $1.500 \mathrm{~g}$ un 19\% (23 pacientes de 120 ingresados) y de $5 \%$ entre los mayores de 1.500 g. (76 pacientes de 1.500 ingresados) ( Figuras 4 y 5 ).

Las complicaciones observadas con mayor frecuencia fueron: EPOC (24\%), neumotórax (21\%), hemorragia intracraneana (21\%), DAP $(14 \%)$, hemorragia pulmonar (10\%) y ROP (1\%) (Figura 6).

La mortalidad de los pacientes que presentaron EMH fue de 33,5\%, distribuida así: los menores de 1.000 gr. tuvieron una mortalidad del $70 \%$; entre 1.0001 y 1.250 g fue de $36 \%$; de $18 \%$ en los niños con un peso de $1.250 \mathrm{~g}$ a $1.500 \mathrm{~g}$ y de $10 \%$ para los mayores de 1.500 g. (Figura 7).

En el estudio, el promedio de días de ventilación mecánica fue de 7 , con una moda de $5 \pm 2$ días y el promedio de estancia hospitalaria fue de 23 días, con una moda de $15 \pm 7$ días.

\section{Dïscusión}

La incidencia de EMH en nuestra institución está acorde con lo descrito en la literatura mundial; comprobando que la población de alto riesgo son los recién nacidos pretérmino menores de $1.000 \mathrm{~g}$.

Tabla 2. Correlación entre el peso y el número y el porcentaje de pacientes con EMH y la población general

\begin{tabular}{|c|c|c|c|c|c|c|c|c|c|c|c|c|c|c|}
\hline $\begin{array}{l}\text { MESI } \\
\text { PESO }\end{array}$ & $1.000 \mathrm{gr}$ & EMH & $\%$ & $\begin{array}{c}1.001 . \\
1.250 \mathrm{gr}\end{array}$ & EMH & $\%$ & $\begin{array}{c}1.251- \\
1.500 \mathrm{gr}\end{array}$ & EMH & $\%$ & $>1.500$ & EMH & $\%$ & TOTAL & $\begin{array}{c}\text { TOTAL } \\
\text { EMH }\end{array}$ \\
\hline Ene-99 & 4 & 2 & 50 & 4 & 1 & 25 & 9 & 1 & 11 & 91 & 3 & 3 & 108 & 7 \\
\hline Feb-99 & 3 & 1 & 33 & 6 & 2 & 33 & 7 & 2 & 29 & 76 & 3 & 4 & 92 & 8 \\
\hline Mar-99 & 4 & 2 & 50 & 3 & 0 & 0 & 8 & 0 & 0 & 91 & 2 & 2 & 106 & 4 \\
\hline May-99 & 1 & 0 & 0 & 6 & 3 & 50 & 3 & 3 & 100 & 95 & 6 & 6 & 105 & 12 \\
\hline Jun-99 & 0 & 0 & 0 & 2 & 2 & 100 & 5 & 2 & 40 & 81 & 2 & 2 & 88 & 6 \\
\hline Jul-99 & 0 & 0 & 0 & 2 & 2 & 100 & 5 & 2 & 40 & 59 & 3 & 5 & 66 & 7 \\
\hline Ago-99 & 4 & 2 & 50 & 3 & 1 & 33 & 6 & 1 & 17 & 65 & 7 & 11 & 78 & 11 \\
\hline Sep-99 & 2 & 1 & 50 & 3 & 2 & 67 & 5 & 2 & 40 & 66 & 4 & 6 & 76 & 9 \\
\hline Oct-99 & 4 & 4 & 100 & 4 & 1 & 25 & 7 & 1 & 14 & 53 & 2 & 4 & 68 & 8 \\
\hline Nov-99 & 1 & 1 & 100 & 4 & 1 & 25 & 4 & 1 & 25 & 54 & 5 & 9 & 63 & 8 \\
\hline Dic-99 & 2 & 2 & 100 & 1 & 0 & 0 & 2 & 0 & 0 & 77 & 3 & 4 & 82 & 5 \\
\hline Feb-00 & 1 & 1 & 100 & 23 & 1 & 4,3 & 7 & 1 & 14 & 33 & 0 & 0 & 64 & 3 \\
\hline Mar-00 & 2 & 2 & 100 & 0 & 0 & 0 & 8 & 0 & 0 & 70 & 5 & 7 & 80 & 7 \\
\hline Abr-00 & 6 & 2 & 33 & 2 & 0 & 0 & 3 & 0 & 0 & 82 & 2 & 2 & 93 & 4 \\
\hline Jun-00 & 2 & 0 & 0 & 2 & 2 & 100 & 7 & 2 & 29 & 62 & 2 & 3 & 73 & 6 \\
\hline Jul-00 & 3 & 2 & 67 & 1 & 0 & 0 & 2 & 0 & 0 & 73 & 3 & 4 & 79 & 5 \\
\hline Ago-00 & 1 & 1 & 100 & 1 & 0 & 0 & 12 & 0 & 0 & 55 & 7 & 13 & 69 & 8 \\
\hline Sep-00 & 1 & 1 & 100 & 3 & 2 & 67 & 5 & 2 & 40 & 69 & 4 & 6 & 78 & 9 \\
\hline Oct-00 & 4 & 4 & 100 & 4 & 1 & 25 & 7 & 1 & 14 & 53 & 2 & 4 & 68 & 8 \\
\hline TOTAL & 49 & 31 & 63 & 79 & 23 & 29 & 120 & 23 & 19 & 1546 & 73 & 5 & 1794 & 150 \\
\hline$\%$ & 100 & 19 & 19 & 100 & 14 & 14 & 100 & 14 & 14 & 100 & 43 & 43 & 100 & 9 \\
\hline
\end{tabular}




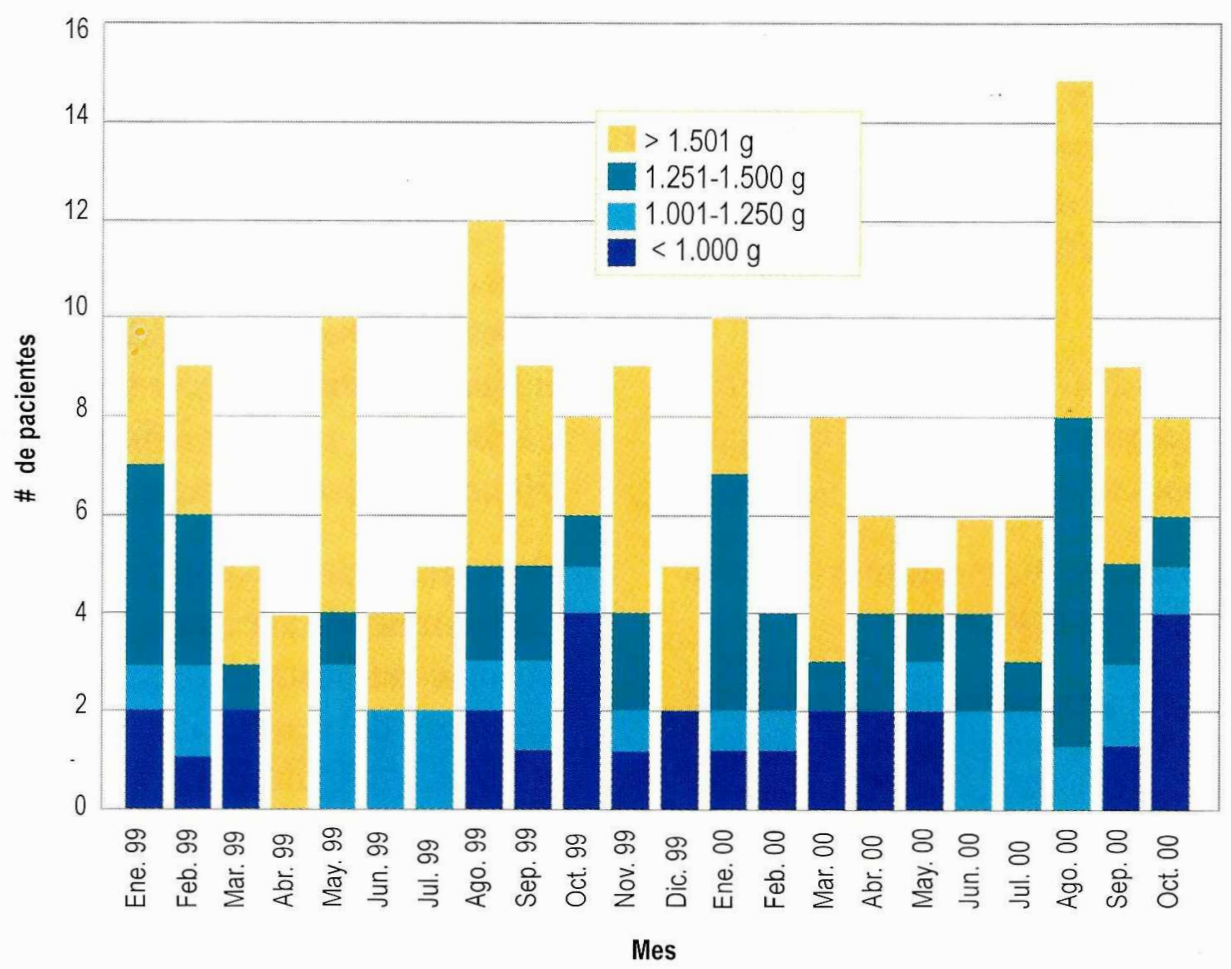

Figura 4. Porcentaje de mortalidad por enfermedad de membrana hialina, según el peso.

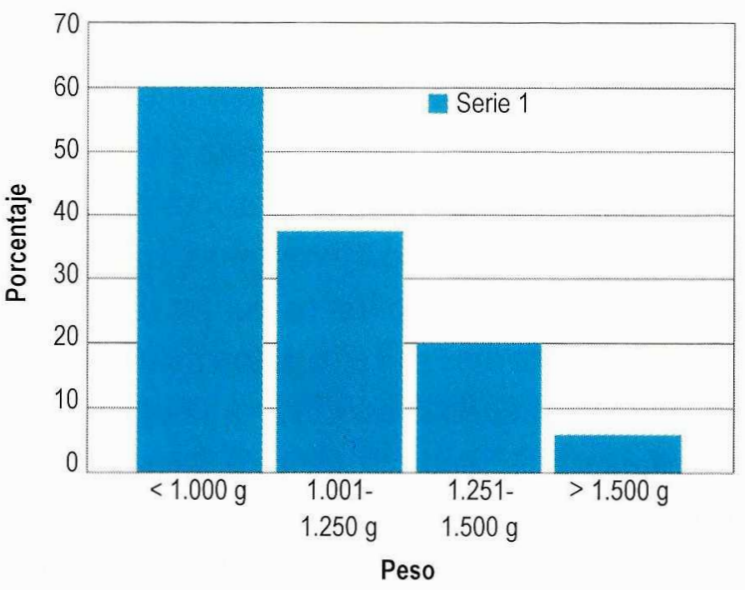

Figura 5. Complicaciones de la enfermedad de mebrana hialina según el número de pacientes.

La complicación observada con mayor frecuencia fue la enfermedad pulmonar crónica del recién nacido en un $24 \%$, seguida de neumotórax en $21 \%$ de los casos y en el recién nacido pretérmino extremo la hemorragia intracraneana con un $17 \%$ y la ROT en un $1 \%$.

La mortalidad encontrada en el servicio (33,5\%) fue mayor respecto a las cifras mundiales, descritas entre $20 \%$ y $30 \%$ y fue aún mayor en los pacientes con un peso inferior a $1.000 \mathrm{~g}$, posiblemente asociada a los riesgos múltiples por la prematurez.

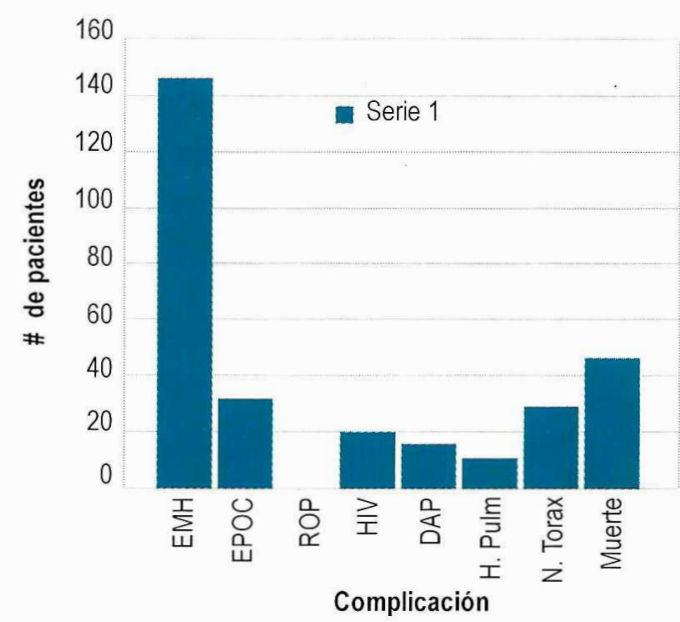

Figura 6. Porcentaje de enfermedad de membrana hialina según el peso.

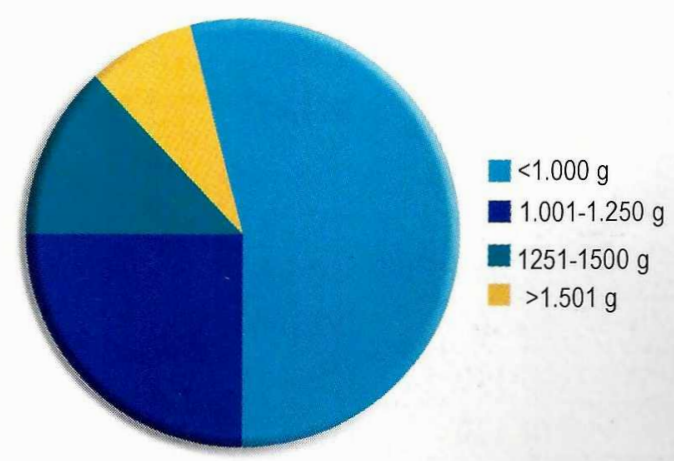

Figura 7. Incidencia de enfemedad de mebrana hialina por mes, según el peso. 
Con el fin de disminuir la morbimortalidad del SDR, así como sus posibles complicaciones y prevenir las estancias hospitalarias prolongadas, hemos planteado las siguientes estrategias, teniendo en cuenta que siempre es fundamental individualizar cada caso:

1. Termorregulación: ideal de $37^{\circ} \mathrm{C}$; monitoreo hemodinámico y respiratorio estricto, respiratorio y accesos venosos adecuados.

2. Uso de corticoides antenatales (mínimo 48 horas antes del parto):

- Betametasona $12 \mathrm{mg}$ intramuscular (IM) cada 24 horas en dos dosis.

- Dexametasona 6mg IM cada 12 horas, por cuatro dosis.

3. Restricción hídrica: El aporte se individualiza dependiendo de la edad gestacional y la edad postnatal (Tabla 3).

4. Cierre profiláctico del DAP: indicado en RNPT menor de $1.000 \mathrm{~g}$ al nacer, menores de 6 horas de vida con recuento de plaquetas mayor de 75.000:

- Indometacina $0,5 \mathrm{mg} / \mathrm{k} /$ dosis intravenoso (IV). Repetir a las 12 horas, posteriormente dos dosis más hasta completar cuatro dosis. Requiere monitoreo estricto del recuento plaquetario y del gasto urinario (mayor de $0,5 \mathrm{cc} / \mathrm{kg} /$ hora), electrolitos séricos, suspender la vía oral hasta 24 horas después de la última dosis. ${ }^{51,52}$ Otras alternativas de manejo tales como el ibuprofeno ofrecen alentadoras esperanzas para el cierre y profilaxis del DAP.

5. Uso de surfactante profiláctico: indicado en menores de 30 semanas o $1.250 \mathrm{~g}$ de peso: se coloca inmediatamente al nacer o una hora después del nacimiento, como máximo. Curosurf® 100 a $200 \mathrm{mg} / \mathrm{kg}(1 \mathrm{cc}=80 \mathrm{mg})$.

6. Prevención de neumonía asociada al ventilador. Se recomienda adecuado lavado de manos; adecuada esterilización y recambio oportuno de los circuitos de ventilación y terapia respiratoria; mínima manipulación; asepsia y antisepsia en los procedimientos a realizar (intubación, cateterizaciones, venopunciones, etcétera); disminución del uso de xantinas y anti-H2; utilización racionalizada y consecuente de los antibióticos de amplio espectro; ventilación en decúbito prono y cambios frecuentes de posición y, por último, correcta ubicación del tubo orotraqueal.

7. Prevención de la infección por Ureaplasma urealyticum: Se han demostrado cultivos de secreción orofaríngea positivos para Ureaplasma urealyticum en 50\% de los pacientes de la UCIN con más de siete días en ventilación mecánica; además, este germen está asociado a la presencia de neumonía y DBP entre la segunda y tercera semana de vida. Por esta razón, en pacientes de muy bajo peso, con ventilación mecánica y deterioro clínico es adecuada la administración de claritromicina, a dosis de $15 \mathrm{mg} / \mathrm{kg} /$ día repartidos en dos dosis cada 12 horas o azitromicina $0,6 \mathrm{mg} / \mathrm{kg} .{ }^{55}$

\begin{tabular}{lcccc}
$\begin{array}{l}\text { Edad } \\
\text { post-natal } \\
\text { Ier día }\end{array}$ & $\begin{array}{c}\text { RNPT } \\
\text { (cc/kg/día) }\end{array}$ & $\begin{array}{c}\text { RNAT } \\
\text { (cc/kg/día) }\end{array}$ & $\begin{array}{c}\text { Sodio } \\
\text { (mEq/kg/día) }\end{array}$ & $\begin{array}{c}\text { Potasio } \\
(\mathbf{m E q} / \mathbf{k g} / \text { día })\end{array}$ \\
\hline $2^{\circ}$ día & $60-70$ & $70-80$ & - & - \\
\hline $3^{\text {er día }}$ & $70-80$ & $80-90$ & 1 & - \\
\hline $4^{\circ}$ día & $80-90$ & $90-100$ & 2 & 1 \\
\hline $5^{\circ}$ día & $100-110$ & $100-110$ & 3 & 1,5 \\
\hline
\end{tabular}

RNPT $=$ Recién nacido pretérmino.

RNAT = Recién nacido a término.
8. $\mathrm{CO}_{2}$ permisivo (45-55) si el $\mathrm{pH}$ es adecuado.

9. Volumen corriente bajo: $3-4 \mathrm{cc} / \mathrm{kg}$. disminuye el riesgo de complicaciones a corto y largo plazo.

10. Prevención de la hemorragia intracraneana: mínima manipulación (evitar movimientos bruscos), utiliza- 
ción de indometacina, sedación idealmente con morfina a dosis de $0,05 \mathrm{mg} / \mathrm{kg}$ o con fentanyl en bolos de 2-4 mcg/kg, utilización de esteroides antenatales, disminuir la frecuencia e intensidad de la terapia respiratoria.

11.Utilización de esteroides tempranos (pacientes menores de 7 días en ventilación mecánica): Dexametasona, 0,2 a $0,5 \mathrm{mg} / \mathrm{kg} /$ día; disminuir la tercera parte cada tercer día hasta suspender.

12.Uso de vitamina A: $2.000 \mathrm{UI} / \mathrm{kg}$ IM interdiarias, por catorce dosis o $4.000 \mathrm{UI}$ vía enteral/día, iniciándose al décimo día de edad post-natal.

13. Ventilación mecánica sincronizada intermitente: su uso disminuye la incidencia de barotrauma y volutrauma, así como la de hemorragia intracraneanal.

14.Apoyo nutricional: iniciación temprana de estímulo enteral $0,5 \mathrm{cc} / \mathrm{kg}$ cada tres horas, con aumento progresivo si las condiciones del paciente lo permiten, hasta lograr un aporte ideal de $65 \mathrm{kcal} /$ $\mathrm{kg} /$ día.

\section{Conclusión}

Durante los últimos meses no se ha visto una disminución en las complicaciones ni en la mortalidad de los pacientes con EMH en la UCIN. Queda la inquietud de realizar un nuevo estudio prospectivo al aplicar las estrategias sugeridas y valorar su utilidad y eficacia para disminuir la morbimortalidad de los recién nacidos que presentan esta patología.

\section{Referenclas}

1. Correa J, Piñeros JG, Gómez A y col. Síndrome de Dificultad Respiratoria del Recién Nacido. UCIN 2000;1: 22-9.

2. Taeusch W, Ballard R. Avery's Diseases of the Newborn. Séptima Edición, W.B. Saunders Company 1998. Pág: 602612.

3. Fanaroff A, Martin R, y col. Neonatal. Perinatal Medicine, Sexta Edición, Editorial Mosby, 1998: 1018-29.

4. Hack M, Very low birthweight outcomes of the NICHD neonatal network November 1989 - October 1990, Am J Obst Gynecol 172:457.

5. Taussing, Linn M, Landau Louis, Pediatric Respiratory Me- dicine, 1999. Editorial Mosby. Primera Edición. Páginas 464468.

6. Tapia J, Ventura P, Manual de Neonatología. Segunda Edición. Editorial Mediterráneo. 2000. Capítulo 27, Páginas 244-247.

7. Lee K, Khoshnood B, Wall S. Trend in mortality from respiratory distrés syndrome in the Unites States, 1.970-1.995. J Ped 1999; 134: 4.

8. Ranganathan D, Wall S y col. Racial differences in respiratory-related neonatal mortality among very low birth weight infants. J Ped 2000;136: 4.

9. Abbasi S, Hirsch D, Davis J. Effect of single versus multiple courses of antenatal corticosteroids on maternal and neonatal outcome. Am Jr of Obstetrics and Gynecology 2000; 182: 5.

10. Van Golde LMG, The pulmonary surfactant system: biochemical aspects and functional significance, Physiol Rew 1988, 68:374.

11. Kattwinkel J, Bloom B, Delmore P. High-versus low trheshold surfactant retreatment for neonatal Respiratory Distrés Syndrome. Pediatrics 2000; 106: 2.

12. Hallman M, Phosphatidylinositol and phosphatidylglycerol in amniotic fluid: indices of lung maturity. Am J Obstet Gynecol 1976; 125:613.

13. Hallman M, Role of myo-inositol in the synthesis of phosphatidylglycerol and phosphatidylinositol in the lung: Biochem Biophys Res Commun 1980; 92:1151.

14. Ikegami M, Surfactant protein A metabolism in preterm vetiled lambs, Am J Physiol 1992; 262:765.

15. Weaver TE, Function and regulation of expression of pulmonary surfactant-associated proteins, Biochem Jr 1991; 273:249.

16. McNeely TB, Comparison of the opsonic activity of human surfactant protein A for Staphylococcus aureus and Streptococcus pneumoniae with rabbit and human macrophages, J Infect Dis 1993; 167:91.

17. O'Brodovich $\mathrm{H}$, Bicarbonate concentration in rhesus monkey and guinea pig fetal lung fluid, Am Rev Respir Dis 1992; 146:1613.

18. Possmayer F: The role of surfactant-associated proteins, Am Rev Respir Dis 1990;142:749.

19. Hamvas A, Surfactant protein B deficiency: antenatal diagnosis and prospective treatment with surfactant replacement, $\mathrm{J}$ Pediatr 1994;125:356.

20. DeMello DE, Molecular and phenotypic variability in the congenital alveolar proteinosis syndrome associated with inherited surfactant protein B deficiency. J Pediatr 1994; 125:43. 21. Clements JA, Kinetics of surface active material in fetal lung. In Hodson WA, editor: Development of the lung, New York, 1977; 543-544.

22. Gluck L, The interpretation and significance of the lecithin/ sphingomyelin ratio in amniotic fluid. Am $\mathbf{J}$ Obstet Gynecol 1974; 120:142.

23. Kulovich MV, The lung profile. Am J Obstet Gynecol 1979; 135:57.

24. Pryhuber GS, Ontogeny of surfactant proteins A and B in human amniotic fluid as indices of fetal lung maturity, Ped Res 1991; 30:597. 
25. Jobe AH, et al: Surfactant for the treatment of respiratory distress syndrome, Am Rev Respir Dis 1987; 136:1256.

26. Hallman M, Surfactant protein A, phosphatidylcoline, and surfactant, inhibitors in epithelial lining fluid. Am Rev Resp Dis 1994; 144:1376.

27. Glatz T, Metabolism of exogenously administered natural surfactant in newborn lambs, Pediatr Res 1982; 16:711.

28. Garland J, Alex C, Pauly T y col. A three day course of dexamethasone therapy to prevent chronic lung disease in ventilated neonates. Pediatrics 1999; 104: 1.

29. Sinkin R, Dweck H, Horgan M y col. Early dexamethason attempting to prevent chronic lung disease. Pediatrcis 2000; 105: 3 .

30. American Academy or Pediatrics. Surfactant replacement therapy for Respiratory Distrés Syndrome. Pediatrics 1999; 103: 3 .

31. Jobe HA, et al:Catabolism and recycling of surfactant: In Robertson B, et al, editors: The pulmonary surfactant system, Amsterdam, 1992.

32. Newman B. Imaging of medical disease of the newborn lung. Radiologic Clinics of North America 1999; 37: 6.

33. Ablow RC, A comparison of early onset group B streptococal neonatal infection and the respiratory distrés syndrome of the newborn. N Engl J Med 1976; 294:65.

34. Mariani G, Cifuentes J, Carlo W. Randomized trial of permissive hypercapnia in preterm infants. Pediatrics 1999; 104: 5 .

35. McEvoy C, Bowling S, Williamson K y col. Timing of antenatal corticosteroids an neonatal pulmonary mechanics. American Journal of Obstetrics and Gynecology 2000; 183: 4. 36. Klaus, Marshall, Fanaroff, Avroy: Care of the high- risk neonate. Novena Edición 1993.

37. Fujiwara T, Artificial surfactant therapy in hyaline membrane disease, Lancet 1980; 1:55.

38. Didziulis L, Perna J, Varela M, Martínez L, Mateus M, Campo M y Col, Uso de Eritropoyetina en Pretérminos, Hospital de San José 1998.

39. Hallman M, Exogenous human surfactant for treatment of severe respiratory distrés syndrome: a randomized prospective clinical trial, J Pediatr 1985;106:963.
40. Phibbs RH, Inictial clinical rial of EXOSURF, a proteinfree synthetic surfactant, for he prophylaxis and early treatment of hyaline membrane disease, Pediatrics 1991; 88:1.

41. Clinicas de Perinatologia. Controversias en el cuidado pulmonar neonatal, Editorial McGraw-Hill 1998, Pág 17-35, 85-90,105-122, 195-220.

42. Yamaguchi N, Togari H, Suzuki S. Apparatus and techniques. Critical Care Medicine 2000; $28: 5$.

43. Clark R, Slutsky A, Gerstmann D. Lung protective strategies of ventilation in the neonate. Pediatrics 2000; 105: 1.

44. Lemons J, Blackmon L, Danto W y col. Use of Inhaled Nitric Oxide. AAP. Pediatrices 2000; 106: 2.

45. Rimensberger P, Beghetti M, Hanquinet $S$ y col. First intention High-frequency Oscillation with early lung volume optimization improves pulmonary outcome in very low birth weight infants with SDR. Pediatrics 2000; 105: 6.

46. Farrell P, Fiascone J. Bronchopulmonary Dysplasia in the 1990s. Curr Probl Pediatr 1997: 133 - 63.

47. Bancalari E. Comentario sobre la Displasia Broncopulmonar. Neonatología Práctica 1998. Tercera Edición: 246-249.

48. Shenai J, Mellen B, Chytil F. Vitamin A Status and Postnatal Dexamethasone Treatment in Bronchopulmonary Dysplasia. Pediatrics 2000, 1-6; 3 .

49. Montenegro M, Amaya L, Retinopatia de la prematurez. Actualizaciones Pediatricas 1998,8; 3:96-100

50. Volpe J, Neurology of the newborn. Tercera ediciòn. Filadelfia: W.B. Saunders, 1995, cap,11.

51. Cloherty J, Stark A. Manual de Cuidados Neonatales. Tercera ediciòn, Barcelona: Editorial Masson 1999, Cap,6,24,2

52. Couser R, Neurodevelopmental follow-up at 36 months 's corrected age of preterm infants treated with prophylactic indomethacin. Arc Pediatr Adolesc Med 2000, 154; 6: 598602

53. Angerio A, Kot P. Closure of the ductus arteriosus new insights for critical care. Critical Care Nursing Quarterly 1998, 20; 4: 80-85.

54. Pacifico L, Panero A, Roggini M. Ureaplasma urealyticum and pulmonary outcome in a neonatal intensive care population. Pediatr Infect Dis J 1997, 16; 579-86. 